\title{
Response to alectinib after one year of discontinuation of crizotinib in anaplastic lymphoma kinase-positive non-small-cell lung cancer: A case report
}

\author{
HIROKO WATANABE ${ }^{1,2}$, TOMOHIRO TAMURA ${ }^{1,2}$, TOSHIHIRO SHIOZAWA $^{2}$, GEN OHARA ${ }^{1}$, \\ KATSUNORI KAGOHASHI $^{1}$, MIO KAWAGUCHI ${ }^{2}$, KOICHI KURISHIMA ${ }^{1}$, \\ HIROAKI SATOH $^{1}$ and NOBUYUKI HIZAWA ${ }^{2}$
}

\author{
${ }^{1}$ Division of Respiratory Medicine, Mito Medical Center, University of Tsukuba, Mito, Ibaraki 310-0015; \\ ${ }^{2}$ Division of Respiratory Medicine, Faculty of Medicine, University of Tsukuba, Tsukuba, Ibaraki 305-8575, Japan
}

Received January 19, 2015; Accepted February 16, 2015

DOI: $10.3892 / \mathrm{mco} .2015 .531$

\begin{abstract}
Therapy with crizotinib achieves prolonged progression-free and overall survival in non-small-cell lung cancer (NSCLC) patients with echinoderm microtubule-associated protein-like 4 (EML4)-anaplastic lymphoma kinase (ALK). It was demonstrated that ALK-positive NSCLCs exhibit a high response rate to the ALK inhibitor, crizotinib. However, a proportion of the patients discontinue crizotinib treatment due to adverse events. This is the case report of a NSCLC patient with EML4-ALK rearrangement, who, following crizotinib discontinuation for one year due to adverse events, exhibited a marked response to alectinib. Even if the incidence is not high, clinicians should not overlook the most common crizotinib-related adverse events. Furthermore, certain patients may continue to benefit from alectinib following long-term discontinuation of crizotinib therapy.
\end{abstract}

\section{Introduction}

The treatment of patients with advanced non-small-cell lung cancer (NSCLC) harboring echinoderm microtubule-associated protein-like 4 anaplastic lymphoma kinase (EML4-ALK) rearrangement has been revolutionized by the development of crizotinib, a small-molecule inhibitor of the receptor tyrosine kinases ALK, ROS1 and MET $(1,2)$. Crizotinib has been reported to be well tolerated; however, a proportion of the patients are unable to continue treatment due to the adverse events (2). Several more potent and highly selective

Correspondence to: Professor Hiroaki Satoh, Division of Respiratory Medicine, Mito Medical Center, University of Tsukuba, 3-2-7, Miyamachi, Mito, Ibaraki 310-0015, Japan

E-mail: hirosato@md.tsukuba.ac.jp

Key words: anaplastic lymphoma kinase-positive non-small-cell lung cancer, adenocarcinoma, alectinib, crizotinib second-generation ALK inhibitors have recently emerged for the treatment of ALK-positive NSCLC, such as alectinib (3). Alectinib appears to exhibit a more favorable toxicity profile compared to crizotinib (4).

This is the case report of a NSCLC patient successfully treated with alectinib, following discontinuation of crizotinib therapy for one year due to adverse events. This case indicates that selected patients may continue to benefit from alectinib following long-term discontinuation of crizotinib therapy.

\section{Case report}

A 41-year-old male patient presented with bilateral cervical and axillar lymph node enlargement. The patient underwent a chest computed tomography (CT) scan, which revealed a nodule in the right upper pulmonary lobe and ipsilateral mediastinal lymph node enlargement. A cervical node biopsy was performed and the histological examination confirmed the diagnosis of adenocarcinoma. Immunohistochemical staining was positive for thyroid transcription factor- 1 and surfactant protein A. The patient had an Eastern Cooperative Oncology Group performance status of 1 and received 4 cycles of carboplatin, pemetrexed and bevacizumab. As the tumor response according to the Response Evaluation Criteria In Solid Tumors (RECIST) guidelines was classified as partial response (PR), the patient subsequently received maintenance chemotherapy with pemetrexed and bevacizumab for 6 cycles. However, the treatment was discontinued due to disease progression.

Written informed consent for the publication of the medical documents was obtained from the patient.

As fluorescence in situ hybridization revealed a translocation of ALK, the patient received crizotinib therapy. After one month, the physical examination and chest $\mathrm{CT}$ revealed a significant decrease in the maximum aggregate tumor measurement. The tumor response was classified as PR, but the treatment was discontinued due to crizotinib-related grade 3 general fatigue, according to the National Cancer Institute-Common Toxicity Criteria, version 2.0. The patient declined additional treatment and all the lesions, including the 


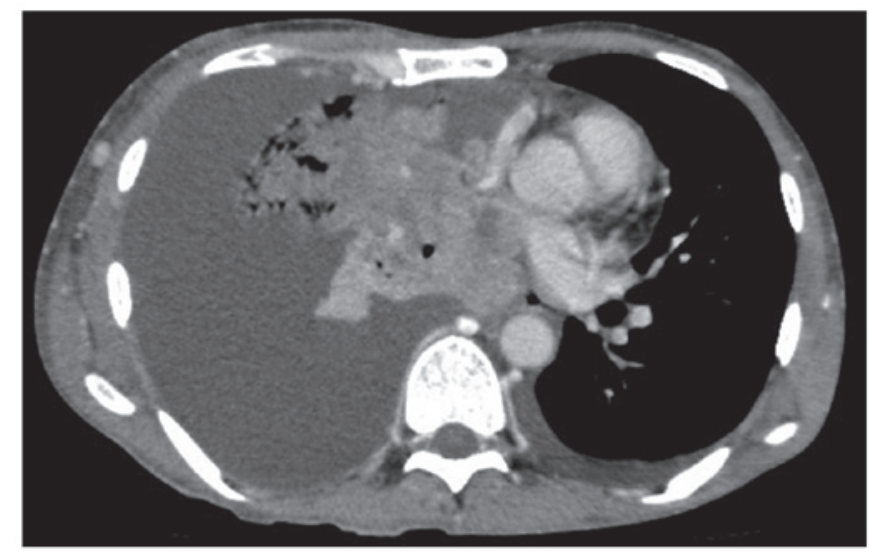

Figure 1. Chest computed tomography (CT) scan showing a large mass with a massive pleural effusion.

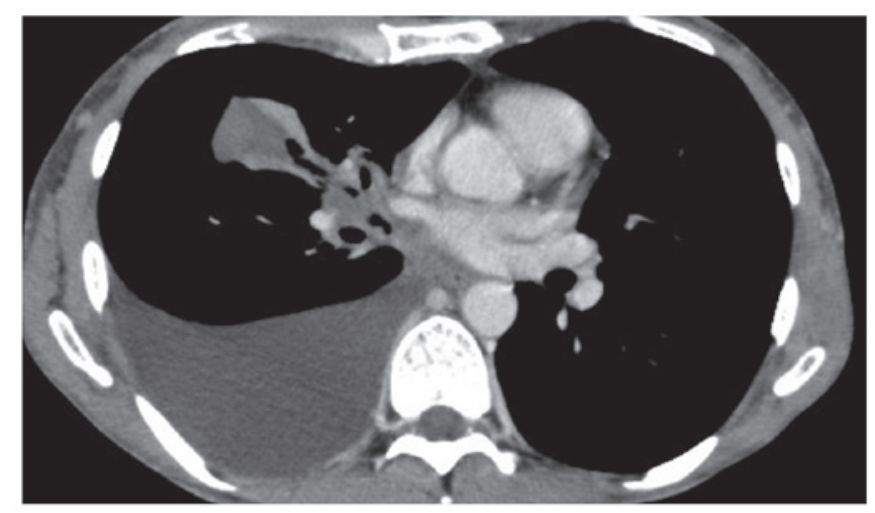

Figure 2. Chest computed tomography (CT) scan 2 weeks after alectinib initiation revealing a significant decrease in the maximum aggregate tumor measurement.

primary site and cervical, axillar, mediastinal and abdominal lymph node metastases, increased in size. A chest CT scan revealed a large mass with a massive pleural effusion (Fig. 1). One year after the discontinuation of crizotinib, the patient consented to receiving alectinib. After 2 weeks of alectinib treatment, a chest $\mathrm{CT}$ revealed a significant decrease in the maximum aggregate tumor measurement (Fig. 2). The tumor response according to the RECIST guidelines was classified as PR. Adverse events, including general fatigue, were not observed. At 4 weeks after the initiation of alectinib, the patient remained alive and adverse event-free.

\section{Discussion}

Gaining genetic insight into the pathogenesis of NSCLC has paved the way for significant advances in its treatment (5). ALK was recently found to exert a potent transforming effect through genetic rearrangement in approximately $5 \%$ of patients with NSCLC (5). Tumor cells with EML4-ALK rearrangement are dependent on its function, similar to tumor cells harboring epithelial growth factor receptor mutations (5). Crizotinib is a first-generation ALK inhibitor, which was previously reported to prolong progression-free survival, increase response rates and improve the quality of life in patients with advanced ALK-positive NSCLC $(6,7)$. However, despite its marked efficacy in patients with ALK-positive NSCLC, crizotinib has been associated with certain adverse events, such as visual disorders, gastrointestinal side effects and elevated liver aminotransferase levels (7). Two recent large-scale phase III studies in patients with advanced AKL-positive NSCLC, with or without previous systemic treatment for advanced disease, demonstrated that the most common adverse events were visual disorders, diarrhea, nausea and edema $(8,9)$. As visual disorders have been specifically associated with crizotinib therapy, attention has been focused on these adverse events, although they are not severe or life-threatening. In a previous study conducted by Blackhall et al (9), comparing crizotinib and chemotherapy in previously treated patients, crizotinib improved symptoms including fatigue, cough, pain and dyspnea, whether the comparator was pemetrexed or docetaxel. General fatigue is not a common toxicity associated with crizotinib therapy (7); however, our patient exhibited grade 3 general fatigue and declined additional therapy. Even if the incidence is not high, clinicians should not overlook the most commonly observed adverse events experienced by the patients.

Alectinib, one of the highly selective second-generation ALK inhibitors, was recently shown to be effective in crizotinib-naive patients, as well as in those resistant to crizotinib (10,11). Common adverse events also associated with alectinib are visual disorders, gastrointestinal side effects and pulmonary toxicity (12). However, the toxicity profile of alectinib has been reported to be milder compared to that of crizotinib (4). To date, our patient has developed no severe adverse event, including general fatigue, which was observed during treatment with crizotinib.

In this study, we presented a case of successful treatment with alectinib in an ALK-positive NSCLC patient, following discontinuation of crizotinib therapy for one year. It is recommended that the patient's disease status and adverse events are closely followed in ALK-positive NSCLC patients who are treated with ALK inhibitors.

\section{References}

1. Landi L and Cappuzzo F: Management of NSCLC: Focus on crizotinib. Expert Opin Pharmacother 15: 2587-2597, 2014.

2. Frampton JE: Crizotinib: A review of its use in the treatment of anaplastic lymphoma kinase-positive, advanced non-small cell lung cancer. Drugs 73: 2031-2051, 2013.

3. Rossi A, Maione P, Sacco PC, Sgambato A, Casaluce F, Ferrara ML, Palazzolo G, Ciardiello F and Gridelli C: ALK inhibitors and advanced non-small cell lung cancer (review). Int J Oncol 45: 499-508, 2014.

4. Gadgeel SM, Gandhi L, Riely GJ, et al: Safety and activity of alectinib against systemic disease and brain metastases in patients with crizotinib-resistant ALK-rearranged non-small-cell lung cancer (AF-002JG): Results from the dose-finding portion of a phase 1/2 study. Lancet Oncol 15: 1119-1128, 2014.

5. Pikor LA, Ramnarine VR, Lam S and Lam WL: Genetic alterations defining NSCLC subtypes and their therapeutic implications. Lung Cancer 82: 179-189, 2013.

6. Kijima T, Takeuchi K, Tetsumoto S, et al: Favorable response to crizotinib in three patients with echinoderm microtubule-associated protein-like 4-anaplastic lymphoma kinase fusion-type oncogene-positive non-small cell lung cancer. Cancer Sci 102: 1602-1604, 2011.

7. Shaw AT, Kim DW, Nakagawa K, et al: Crizotinib versus chemotherapy in advanced ALK-positive lung cancer. N Engl J Med 368: 2385-2394, 2013. 
8. Solomon BJ, Mok T, Kim DW, et al; PROFILE 1014 Investigators: First-line crizotinib versus chemotherapy in ALK-positive lung cancer. N Engl J Med 371: 2167-2177, 2014.

9. Blackhall F, Kim DW, Besse B, et al: Patient-reported outcomes and quality of life in PROFILE 1007: A randomized trial of crizotinib compared with chemotherapy in previously treated patients with ALK-positive advanced non-small-cell lung cancer. Thorac Oncol 9: 1625-1633, 2014.

10. Wu YL, Zhou C, Hu CP, et al: Afatinib versus cisplatin plus gemcitabine for first-line treatment of Asian patients with advanced non-small-cell lung cancer harbouring EGFR mutations (LUX-Lung 6): An open-label, randomised phase 3 trial. Lancet Oncol 15: 213-222, 2014.
11. Majem $\mathrm{M}$ and Pallarès $\mathrm{C}$ : An update on molecularly targeted therapies in second- and third-line treatment in non-small cell lung cancer: Focus on EGFR inhibitors and anti-angiogenic agents. Clin Transl Oncol 15: 343-357, 2013.

12. Köhler J and Schuler M: LUX-Lung 3: Redundancy, toxicity or a major step forward? Afatinib as front-line therapy for patients with metastatic EGFR-mutated lung cancer. Future Oncol 10: 533-540, 2014. 